Lepr. Rev. (1977) 48, 129-132

\title{
Primary Dapsone-resistant Leprosy
}

\author{
J. M. H. PEARSON, G. S. HAILE \\ Medical Research Council Leprosy Project, POB 1005, Addis A baba Leprosy Hospital, Ethiopia
}

and

\author{
R. J. W. REES*
}

National Institute for Medical Research, London NW7 1 A A, England

\begin{abstract}
Persons living in areas where acquired dapsone resistance is common may be infected and develop leprosy with dapsone-resistant strains of Mycobacterium leprae. Mouse foot-pad tests on bacilli from 8 such patients, with active and previously untreated lepromatous leprosy, have shown that strains from 5 were dapsone resistant. These findings demonstrate for the first time the presence of patients with primary dapsone resistant leprosy in the community at large. The preliminary findings are presented because of the high proportion of primary dapsone resistance in the first 8 patients in a survey of some 50 patients in Ethiopia. The implications of these preliminary findings are discussed.
\end{abstract}

\section{Introduction}

As the number of lepromatous patients with acquired dapsone-resistant leprosy increases, the likelihood that they will become the source of new cases showing primary dapsone resistance, also increases. However, primary dapsone-resistant leprosy can only be diagnosed by dapsone-sensitivity testing using the mouse foot-pad infection, unless the degree of resistance is so high that patients fail to show any response to dapsone therapy. We report here the results of mouse foot-pad sensitivity tests performed in 8 patients with previously untreated lepromatous leprosy.

\section{Patients and Methods}

Patients were selected from those attending the Addis Ababa Leprosy Hospital. All had active lepromatous leprosy and denied previous treatment. All had lived for at least 5 years in areas where anti-leprosy treatment with mainly dapsone had been available for 10 years or more. A biopsy was taken from an active skin lesion from each patient and transported by air on wet ice to England. The dapsone-sensitivity testing, using the mouse foot-pad infection, was performed at the National Institute for Medical Research, London, not more than 5 days after the biopsy of skin was taken in Ethiopia. The skin was homogenized for the

\footnotetext{
* Requests for reprints should be addressed to R.J.W.R. at the National Institute for Medical Research, London NW7 1 AA, England.

Received for publication 19 February, 1977.
} 
preparation of suspensions of $M$. leprae for the inoculation of both hind foot-pads of mice by the standard procedures previously described (Rees, 1964). For assessment of the dapsone sensitivity of the strains of $M$. leprae the inoculated mice were divided into groups of 6 animals. One group acted as a control and the other group or groups received dapsone incorporated in different concentrations in their diet. Strains of M. leprae from the first 4 patients were screened against only a concentration of $0.0001 \%$ dapsone in the diet whereas the later 4 strains were screened against $0.001,0.0001$ and $0.00003 \%$ dapsone in the diet, see Table 1 (Rees, 1967; Pearson, Rees and Waters, 1975).

\section{Results}

Of the 8 patients only 3 had strains of $M$. leprae that were fully sensitive to dapsone, in that their growth was completely inhibited in mice receiving $0.0001 \%$ dapsone in the diet. The levels of dapsone resistance of the strains from the remaining 5 patients are summarized in Table 1. Of the 3 resistant strains more

TABLE 1

Multiplication of 8 strains of Mycobacterium leprae in mice receiving different concentrations of dapsone in the diet

\begin{tabular}{|c|c|c|c|c|c|}
\hline \multirow[t]{2}{*}{ Patient } & \multicolumn{4}{|c|}{$\begin{array}{c}\text { Bacillary multiplication } \\
\text { \% Dapsone in diet }\end{array}$} & \multirow[t]{2}{*}{$\begin{array}{c}\text { Dapsone } \\
\text { sensitivity }\end{array}$} \\
\hline & 0 & 0.00003 & 0.0001 & 0.001 & \\
\hline 1 & + & - & + & - & Resistant \\
\hline 2 & + & - & 0 & - & Sensitive \\
\hline 3 & + & - & + & - & Resistant \\
\hline 4 & + & - & 0 & - & Sensitive \\
\hline 5 & + & + & 0 & 0 & Sensitive \\
\hline 6 & + & + & + & + & Resistant \\
\hline 7 & + & + & + & + & Resistant \\
\hline 8 & + & + & + & 0 & Resistant \\
\hline
\end{tabular}

broadly screened, 2 showed a higher degree of resistance (i.e. to $0.001 \%$ dapsone in the diet), the third was resistant to only $0.0001 \%$ dapsone in the diet, as were the 2 other dapsone resistant strains tested only at this concentration.

\section{Discussion}

Resistance to dapsone may be either acquired or primary, as is the case with any other drug or micro-organism. Acquired dapsone resistance occurs as a result of the selective multiplication of spontaneous drug-resistant mutant bacilli during the course of dapsone therapy. It is likely to occur more commonly when dapsone dosage is sub-optimal (Pearson et al., 1975), but has been recorded in patients who have been prescribed dapsone in full dosage and taken treatment regularly (Pearson et al., 1975). Primary resistance, on the other hand, implies that the bacilli which infected the patient were dapsone resistant from the beginning. 
Acquired dapsone resistance is now commonly seen, and is becoming a major problem for many leprosy control services. In Addis Ababa, for example, the incidence of suspected acquired dapsone resistance is about $3 \%$ per annum among patients already under treatment for lepromatous leprosy (Pearson, Ross and Rees, 1976). However, this study is the first reported systematic attempt to look for primary dapsone-resistant leprosy, and the fact that bacilli from 5 out of the first 8 newly-diagnosed patients with lepromatous leprosy tested showed dapsone resistance indicates that primary resistance in their home areas may now be the norm rather than an occasional exception.

All the patients tested were suffering from lepromatous leprosy. Dapsone resistance in non-lepromatous ("paucibacillary") cases has not yet been reported, and will only be diagnosable on clinical grounds (though occasionally biopsies might contain enough bacilli for mouse foot-pad tests to be undertaken). However, all leprosy patients are likely to derive their infections from the same index cases. It is therefore probable that, in areas where primary dapsone-resistant lepromatous leprosy is found, non-lepromatous cases will also often be dapsone resistant.

In this survey of primary dapsone resistance we have defined a resistant strain of $M$. leprae as being one capable of multiplying in the foot-pads of mice receiving dapsone at a concentration of $0.0001 \%$. This is based on our own extensive data, and that of others (Levy and Peters, 1976), that all strains of M. leprae from apparently previously untreated patients, from many different parts of the world, were sensitive to this or lower concentrations of dapsone. Therefore when we started the survey strains of $M$. leprae from the first 4 patients were tested only in mice receiving $0.0001 \%$ dapsone, although all subsequent patients, including patients 5 to 8 in this paper, were in addition tested at $0.00003 \%$ and $0.001 \%$ dapsone. The extended assay was introduced, in part, to check the dapsone sensitivity of strains of $M$. leprae from new lepromatous patients currently arising in Ethiopia but also, and importantly, to determine the degree of resistance in patients showing primary dapsone resistance. Since only one of the 3 dapsone resistant strains that were checked showed low grade resistance (to $0.0001 \%$ dapsone), the 2 resistant strains not checked above $0.0001 \%$ dapsone might, unfortunately, also have a higher grade resistance. Low grade resistance determined in the mouse is equivalent to failure to respond to dapsone $1 \mathrm{mg}$ daily in man (Shepard, 1973). Most such patients have responded only for a few years when treated with dapsone in maximal dosage, though occasionally more prolonged remissions occur. .It is likely therefore that most patients with lepromatous leprosy who show even low grade primary dapsone resistance will not be cured by monotherapy with dapsone, even in maximal dosage. It is possible that supplementation with a second drug would be curative; but it is not unlikely that triple therapy will be needed for these cases, at least during an initial period of intensive therapy. It is our experience that patients with higher grade resistance (to $0.001 \%$ dapsone) respond only for a year or perhaps two when treated with dapsone in maximal dosage.

In areas where cases of primary dapsone resistant lepromatous leprosy are common, it must be assumed that the non-lepromatous cases are also resistant. Because of their lower bacterial load, it is more likely that such patients would be cured with dapsone alone, and probable that dual therapy will suffice. Certainly it would be advisable as a minimum measure, to use dual therapy for all leprosy cases in such areas. 
The finding of primary dapsone resistance on a significant scale in Ethiopia has several important implications:

(1) Experimental chemotherapy is needed to determine optimal drug regimens for the treatment as well as the prevention of drug resistant leprosy. Such trials will probably require armadillos infected with sensitive or resistant strains of M. leprae.

(2) Surveys using mouse foot-pad tests of patients in other parts of the world with previously untreated lepromatous leprosy should be planned. It is unlikely that primary dapsone resistance is confined to Ethiopia, and the extent of the problem must be determined.

(3) Multiple drug therapy must become routine practice in leprosy control programmes: this presents a challenge for budgeting and staff training. Furthermore, large scale controlled drug trials will be required if the cost effectiveness of different drug regimens is to be accurately determined.

(4) It is likely that treatment regimens for leprosy should vary according to the risk of dapsone resistance in any particular area. This could be an argument for retaining leprosy as a specialized service, at least until more is known of the problems of chemotherapy, which are only now coming to light for leprosy. Correct anti-leprosy treatment is no longer either simple or cheap, and ill-advised integration could turn a problem into a disaster for the future of leprosy control.

\section{Acknowledgements}

We are grateful to the staff of the Addis Ababa Leprosy Hospital and the All Africa Leprosy and Rehabilitation Training Centre (ALERT) for referral of patients and provision of facilities for investigation and treatment. We are indebted to Mrs Susan Cate and Miss Gillian Cooper for technical assistance in the studies carried out at the National Institute for Medical Research, London.

\section{References}

Levy, L. and Peters, J. H. (1976). Susceptibility of Mycobacterium leprae to dapsone as a determinant of patient response to acedapsone. Antimicrob. Agents Chemother. 9, 102.

Pearson, J. M. H., Rees, R. J. W. and Waters, M. F. R. (1975). Sulphone resistance in leprosy. A review of 100 proven clinical cases. Lancet ii, 69.

Pearson, J. M. H., Ross, W. F. and Rees, R. J. W. (1976). DDS resistance in Ethiopia-a progress report. Int. J. Lepr. 44, 140.

Rees, R. J. W. (1964). Limited multiplication of acid fast bacilli in foot-pads of mice inoculated with Mycobacterium leprae. Brit. J. Exper. Path. 45, 207.

Rees, R. J. W. (1967). Drug resistance of Mycobacterium leprae particularly to DDS. Int. J. Lepr. 35, 625.

Shepard, C. C. (1973). Experimental chemotherapy in leprosy, then and now. Int. J. Lepr. 41, 307. 